Spin-Resolved Electronic Structure Studies of Non-Magnetic Systems: Possible Observation of the Fano Effect in Polycrystal Ce

J.G. Tobin, S.A. Morton, B.W. Chung, S.W. Yu, G.D. Waddill

June 7, 2005

The International Conference on Strongly Correlated Electron Systems

Vienna, Austria

June 20, 2005 through June 30, 2005 
This document was prepared as an account of work sponsored by an agency of the United States Government. Neither the United States Government nor the University of California nor any of their employees, makes any warranty, express or implied, or assumes any legal liability or responsibility for the accuracy, completeness, or usefulness of any information, apparatus, product, or process disclosed, or represents that its use would not infringe privately owned rights. Reference herein to any specific commercial product, process, or service by trade name, trademark, manufacturer, or otherwise, does not necessarily constitute or imply its endorsement, recommendation, or favoring by the United States Government or the University of California. The views and opinions of authors expressed herein do not necessarily state or reflect those of the United States Government or the University of California, and shall not be used for advertising or product endorsement purposes. 


\title{
Spin-Resolved Electronic Structure Studies of Non-Magnetic Systems: Possible Observation of the Fano Effect in Polycrystal Ce
}

\author{
J.G. Tobin ${ }^{1, *}$, S.A. Morton ${ }^{1,2}$, B.W. Chung ${ }^{1}$, S.W. Yu ${ }^{1}$ and G.D. Waddill ${ }^{2}$, \\ 1.Lawrence Livermore National Laboratory, Livermore, CA, USA \\ 2.University of Missouri-Rolla, Rolla, MO, USA \\ ${ }^{*}$ Contact Author: Tobin1@LLNL.Gov
}

\section{Introduction}

The valence electronic structure and electron spectra of Cerium remain a subject of uncertainty and controversy. Perhaps the best and most direct method of ascertaining the valence electronic structure is the application of electron spectroscopies [1-17], e.g. photoelectron spectroscopy for the occupied states [1-10, 12-14] and $x$-ray absorption [2] and Bremstrahlung Isochromat Spectroscopy (inverse photoelectron spectroscopy) $[3,11,13]$ for the unoccupied states. Much of the controversy revolves around the interpretation of the $\mathrm{Ce}$ photoemission structure in terms of a modified Anderson Impurity Model $[15,16]$. Here, in this correlated and multi-electronic picture, semi-isolated $4 \mathrm{f}$ states (at a nominal binding energy of $1 \mathrm{eV}$ ) are in contact with the bath of spd valence electrons, generating spectral features at the Fermi Level and at a binding energy corresponding to the depth of the bath electron well, about $2 \mathrm{eV}$ below the Fermi Level in the case of $\mathrm{Ce}$. This controversy has spilled over into issues such as the volume collapse associated with the alpha to gamma phase transition [17-19] and the electronic structure of Ce compounds [20-23]. (A more generalized schematic illustrating the competition between the bandwidth (W) and correlation strength $(U)$ is shown in Figure 1.) Considering the remaining uncertainty associated with the spectral features and valence electronic structure of $\mathrm{Ce}$, it seemed plausible that the situation would benefit from the application of a spectroscopy with increased resolution and probing power. To this end, we have applied circularly polarized soft $x$-rays and true spin detection, in a modified form of the photoelectron spectroscopy experiment, to the enigmatic Ce system. The result of this is that we have observed the first evidence of the Fano Effect in the valence electronic features of non-magnetic Cerium ultra-thin films.

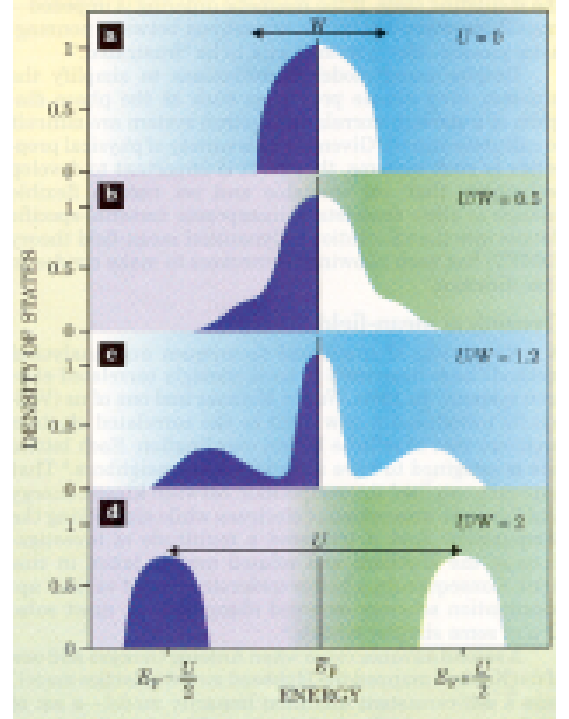

Figure 1 Illustration of the origin of the quasiparticle ( at the Fermi Level, $\mathrm{E}_{\mathrm{F}}$ ) and the Hubbard Bands (at $\pm \mathrm{U} / 2$, relative to the Fermi Level). $W$ is the band width and $U$ is the correlation strength. Case c, third from the top, is the case closest to $\mathrm{Ce}$. Taken from Ref 24. 
The Fano Effect is the observation of spin specific photoelectron emission from the valence bands of a non-magnetic material due to excitation with circularly polarized light. First predicted in 1969 by Fano [25], the effect was experimentally confirmed by measuring the polarization of alkali vapor beams using detection of ions [26-28] and photoelectron emission [29] shortly thereafter. Subsequently, the effect was observed in the spin resolved photoemission of non-alkali systems, including the heavy atoms such as Th [30], $\mathrm{Hg}$ [31], and $\mathrm{Xe} / \mathrm{Pd}$ (111) [32]. A variation of the Fano effect, in the core level photoemission of non-magnetic materials, has also been measured using both circular dichroism [33] and linear dichroism [34,35]. Our data for Au is shown in Figure 2.

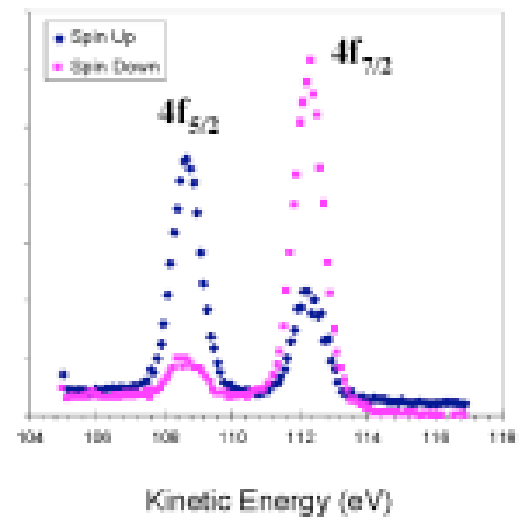

Figure 2

"Double Polarization" spectrum of $\mathrm{Au}(111) 4 \mathrm{f}$ peaks. Note that the combination of circularly polarized excitation with spin polarized detection can be used to produce spin polarized data from non-magnetic sample. The photon energy was $200 \mathrm{eV}$.

\section{Experimental and Preliminary Results}

A new compact angle resolving spin spectrometer for conducting double polarization experiments has recently been developed at the Advanced Light Source. This spectrometer combines a large (11 inch) diameter fixed hemispherical analyzer with a novel rotatable input lens system allowing data with +-1 degree angular resolution to be acquired for any combination of incident and emission angles, including normal incidence / normal emission (Figures 3 \& 4). The analyzer is equipped with both multichannel detection for high resolution $(50 \mathrm{meV})$ spin integrated spectroscopies, such as XPS and magnetic linear or circular dichroism, and a Mott detector capable of resolving the photoelectron spin polarization simultaneously along the two perpendicular axes of the rotational plane. Rapid switching between spin integrated and spin resolved modes is achieved by focusing the photoelectrons through a small hole in the detector of the hemispherical analyzer and into a compact mini-Mott detector situated immediately behind the channelplates.

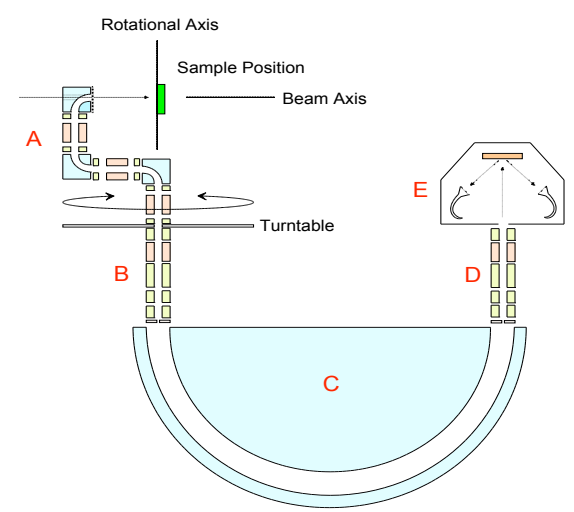

Figure 3

Schematic of the analyzer and lens assembly. (A) Rotating entrance lens assembly. (B)Transfer lenses. (C)Fixed large diameter hemispherical analyzer.

(D)Transfer lenses. (E)Fixed spin resolving Mott detector. 


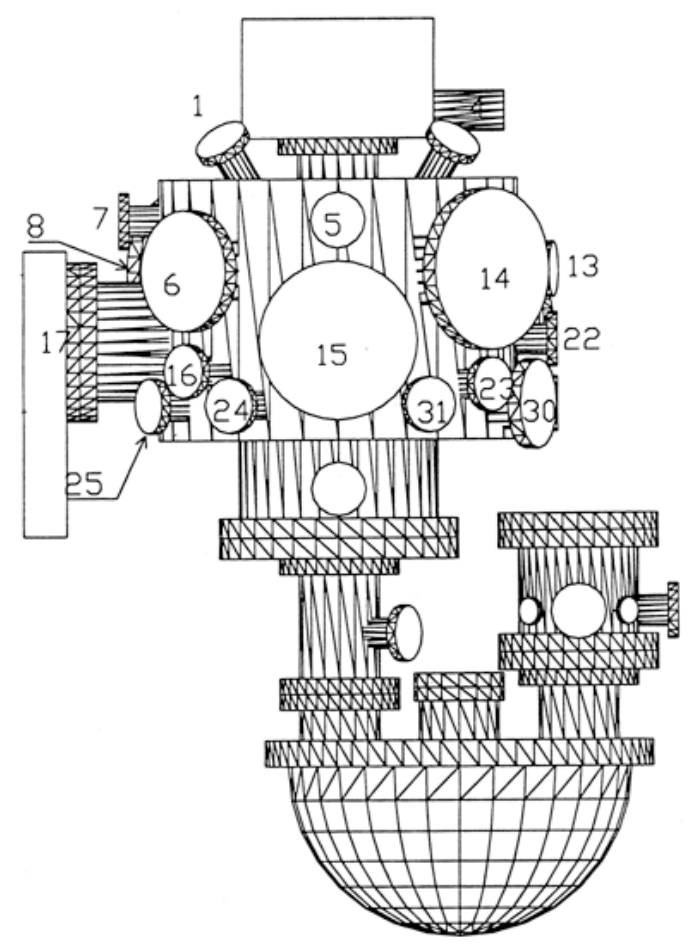

Figure 4

Schematic of the analyzer system mounted beneath the analysis chamber. The resulting instrumental footprint is smaller than most conventional XPS systems.

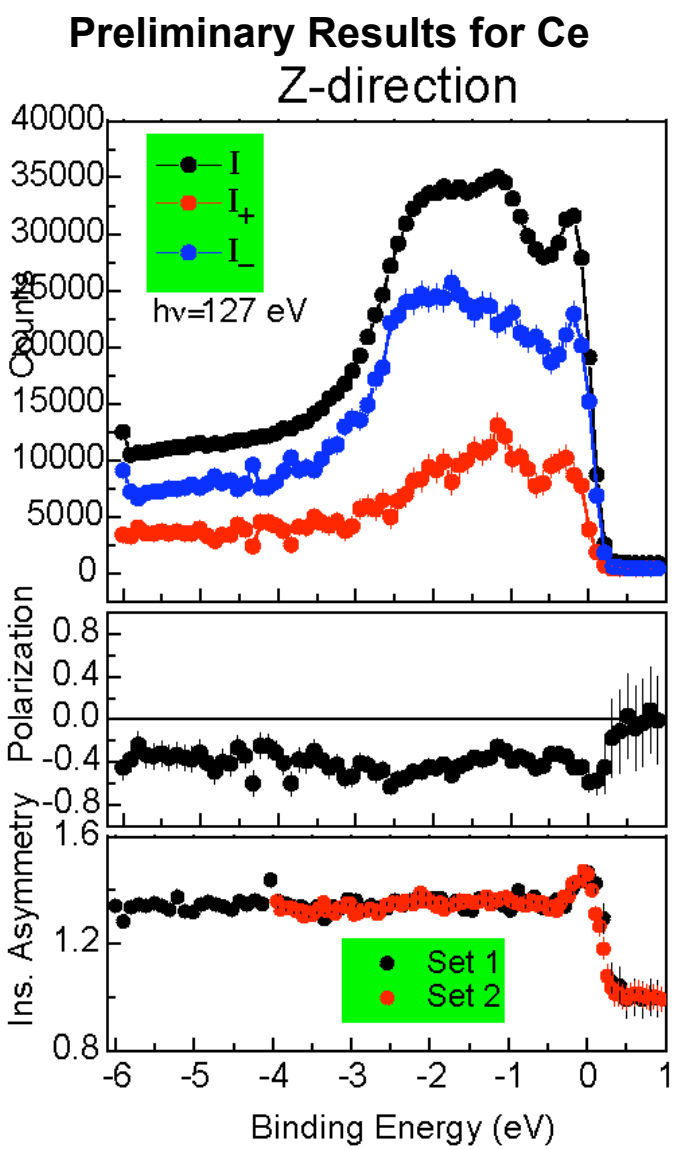

Figure 5

Spin resolved spectra of polycrystalline films of Ce deposited upon W(110). The photon energy was $127 \mathrm{eV}$.

Using circularly polarized $x$-rays and true spin detection via a MiniMott Detector, evidence of strong spin specific effects have been observed in the valence bands of ultrathin films of nonmagnetic Ce. (Fig 5) There is both a large static spin polarization across the entire valence band and a smaller oscillation in the vicinity of the lower Hubbard Band, near binding energies of $1-3 \mathrm{eV}$. The lower Hubbard band may have been broadened by stresses inside the Ce film. These preliminary measurements need to be repeated and extended to other photon energies.

\section{Future Plans}

Using these manifestations of the Fano Effect, the nature of the $\mathrm{Ce}$ and $\mathrm{Pu}$ valence electronic structure will be probed, including the possibility of Kondo shielding in the peak at the Fermi energy.

\section{Acknowledgements}

This work was performed under the auspices of the U.S. DOE by University of California Lawrence Livermore National Laboratory under contract W-7405-Eng48. The ALS has been built and operated under funding from the Office of Basic Energy Science at DOE. 


\section{References}

1. Y. Baer and G. Busch, Phys. Rev. Lett. 31, 35 (1973).

2. D. Wieliczka, J.H. Weaver, D.W. Lynch, and C.G. Olson, Phys. Rev. B 26, 7056 (1982).

3. E. Wuilloud, H.R. Moser, W.D. Schneider, and Y. Baer, Phys. Rev. B 28, 7354 (1983).

4. D.M. Weiliczka, C.G. Olson, and D.W. Lynch, Phys. Rev. B 29, 3028 (1984).

5. D.M. Weiliczka, C.G. Olson, and D.W. Lynch, Phys. Rev. Lett. 52, 2180 (1984).

6. $\quad$ E. Jensen and D.M. Weiliczka, Phys. Rev. B 30, 7340 (1984).

7. F. Patthey, B. Delley, W.D. Schneider, and Y. Baer, Phys. Rev. Lett. 55, 1518 (1985).

8. C. Laubschat, E. Weschke, C. Holtz, M. Domke, O. Strebel, and G. Kaindl, Phys. Rev. Lett. 65, 1639 (1990).

9. F. Patthey, J.M. Imer, W.D. Schneider, H. Beck, Y. Baer, and B. Delley, Phys. Rev. B 42, 8864 (1990).

10. E.Weschke, C. Laubschat, T. Simmons, M. Domke, O. Strebel, and G. Kaindl, Phys. Rev. B 44, 8304 (1991).

11. Y. Baer, M. Grioni, D. Malterre, and W.D. Schneider, Phys. Rev. B 44, 9108 (1991).

12. J.J. Joyce, A.J. Arko, J. Lawrence, P.C. Canfield, Z. Fisk, R.J. Bartlett, and J.D. Thompson, Phys. Rev. Lett. 68, 236 (1992).

13. L.Z. Liu, J.W. Allen, O. Gunnarsson, N.E. Christensen, and O.K. Anderson, Phys. Rev. B. 45, 8934 (1992).

14. E. Weschke, A. Hoehr, G. Kaindl, S. L. Molodstov, S. Danzenbaecher, M. Richter and C. Laubschat, Phys. Rev. B 58, 3682 (1998).

15. O. Gunnarsson and K. Schoenhammer, Phys. Rev. Lett 50, 604 (1983).

16. O. Gunnarsson and K. Schoenhammer, Phys. Rev. B 28, 4315 (1983).

17. J.W. Allen and J.Z. Liu, Phys. Rev. B 46, 5047 1992).

18. B. Johansson, I.A. Abrikosov, M. Alden, A.V. Ruben, and H.L. Skriver, Phys. Rev. Lett. 74, 2335 (1995).

19. K. Held, A.K. McMahan, and R.T. Scarlettar, Phys. Rev. Lett. 87, 276404 (2001).

20. E. Weschke, C. Laubschat, R. Ecker,A. Hoehr, M. Domke, G. Kaindl, L. Severin and B. Johansson, , Phys. Rev. Lett. 69, 1792 (1992).

21. D. Malterre, M. Grioni, Y. Baer, L. Braichovich, L. Duo, P. Vavassori, and G.L. Olcese, Phys. Rev. Lett. 73, 2005 (1994).

22. E. Weschke, C. Laubschat,A. Hoehr, M. Domke, G. Kaindl, L. Severin and B. Johansson, , Phys. Rev. Lett. 73, 2006 (1994).

23. A.J. Arko, J.J. Joyce, A.B. Andrews, J.D. Thompson, J.L. Smith, D. Mandrus, M.F. Hundley, A.L. Cornelius, E. Moshopoulou, Z. Fisk, P.C. Canfield, and A. Menovsky, Phys. Rev. B 56, R7041 (1992).

24. G. Kotliar and D. Vollhardt, Physics Today 57, 53 (March 2004).

25. U. Fano, Phys. Rev. 178, 131 (1969); 184, 250 (1969).

26. M.S. Lubell and W. Raith, Phys. Rev. Lett. 23, 211 (1969).

27. J. Kessler and J. Lorenz, Phys. Rev. Lett. 24, 87 (1970).

28. G. Baum, M.S. Lubell, and W. Raith, Phys. Rev. Lett. 25, 267 (1970).

29. U. Heinzmann, J. Kessler, and J. Lorenz, Phys. Rev. Lett. 25, 1325 (1970).

30. U. Heinzmann, H. Heuer, and J. Kessler, Phys. Rev. Lett. 34, 441 (1975); 34, 710 (1975).

31. Schoenhense, U. Heinzmann, J. Kessler, and N.A. Cherepkov, Phys. Rev. Lett. 48, 603 (1982).

32. B.Vogt, B. Kesser, N. Mueller, G. Schoenhense, B. Schmiedeskamp, U. Heinzmann, Phys. Rev. Lett. 67, 1318 (1991).

33. K. Starke, A. P. Kaduwela, Y. Liu, P. D. Johnson, M. A. Van Hove, C. S. Fadley, V. Chakarian, E. E. Chaban, G. Meigs, and C. T. Chen, Phys. Rev. B 53, R10544 (1996).

34. C. Roth, F.U. Hillebrecht, W.G. Park, H.B. Rose, and E. Kisker, Phys. Rev. Lett. 73, 1963 (1994).

35. H.B. Rose, A. Fanelsa, T. Kinoshita, C. Roth, F.U. Hillebrecht, and E. Kisker, Phys. Rev. B. 53, 1630 (1996). 